\title{
Toxicological screening in trauma
}

\author{
T D Carrigan, H Field, R N Illingworth, P Gaffney, D W Hamer
}

Accident and Emergency Department, St James's University Hospital, Leeds T D Carrigan R N Illingworth P Gaffney

Leeds Teaching Hospitals Chemical Pathology Department H Field

Accident and Emergency Department, Leeds General Infirmary D W Hamer

Correspondence to: Dr T D Carrigan, Specialist Registrar in Accident and Emergency, St James's University Hospital, Beckett Street, Leeds LS9 7TF

Accepted 14 September 1999

\begin{abstract}
Objectives-To determine the prevalence and patterns of alcohol and drug use in patients with major trauma.

Methods-Consecutive trauma patient enrolment, 24 hours a day, was envisaged with anonymised patient data on gender, age band, and mechanism of injury collected. The study group had surplus plasma quantitatively analysed for ethanol concentration, and urine samples were initially screened, via immunoassay, for opiates, cannabinoids, amphetamines, benzodiazepines, cocaine, and methadone. Confirmation and specification of individual positive results was then performed using thin layer or gas-liquid chromatography. Drugs of treatment given in the resuscitation room, if subsequently detected in the urine samples, were excluded from the final results.
\end{abstract}

Results-There were 116 eligible trauma patients assessed and treated in the resuscitation room over a six month period, of which $93(80 \%)$ were enrolled. Altogether $27 \%$ of this trauma population had plasma ethanol concentrations greater than 80 $\mathrm{mg} / \mathrm{dl}$. There was a significantly higher prevalence of alcohol intoxication in the group not involved in a road traffic accident (RTA) compared with the group who were involved in a RTA.

Initial screening of urine for drugs revealed a prevalence of $51 \%$. After 12 exclusions due to iatrogenic administration of opiates, the final confirmed prevalence was $35 \%$ in this trauma population. The individual drug prevalence was $13 \%$ for cannabinoids, $11 \%$ for codeine, $8 \%$ for morphine, $6 \%$ for amphetamine, $6 \%$ for benzodiazepines, $3 \%$ for cocaine, $1 \%$ for dihydrocodeine, and $1 \%$ for methadone.

Conclusions-There is a notable prevalence of drug and alcohol use in this British accident and emergency trauma population.

A significantly higher prevalence for alcohol intoxication was found in the nonRTA group compared with the RTA group.
The patterns of drug usage detected reflect local influences and less cocaine use is seen compared with American studies. The association between alcohol, drugs, and trauma, together with ethically acceptable methods of screening, are discussed.

(F Accid Emerg Med 2000;17:33-37)

Keywords: toxicology; alcohol; substance abuse; trauma

Drug intoxication is associated with trauma, be it interpersonal, personal, or vehicular trauma. There is a clear dose-effect relationship between alcohol and road traffic accidents (RTAs), ${ }^{1}$ but while cannabinoids and central nervous system stimulants and depressants have been shown to influence psychomotor control, ${ }^{2-4}$ causality between both licit and illicit drugs and trauma is multifactorial. ${ }^{5}$ Such factors include age, fatigue, personality traits, medical conditions, and environmental conditions. It could be argued that accident and emergency (A\&E) doctors have a medical and societal responsibility to detect reversible influences such as alcohol and drug intoxication, as studies have shown that such intoxication is associated with recurrent trauma, ${ }^{67}$ future RTA fatalities, ${ }^{8}$ and shelter from culpability. ${ }^{9}$

Different approaches have been made to this problem, including toxicological screening and self report questionnaires. Previous toxicological studies included prevalence studies in seriously injured trauma patients presenting to a trauma centre, ${ }^{1011}$ trauma patients with altered levels of consciousness, ${ }^{12}$ drivers under arrest, ${ }^{13}$ and RTA fatalities. ${ }^{14}$ The prevalence rates of a selected number of applicable studies, including one adolescent study, ${ }^{15}$ are shown in table 1.

In the United States, both the American College of Emergency Physicians and the American College of Surgeons recommend routine urine toxicological screening of trauma victims admitted to trauma centres. ${ }^{17} \mathrm{We}$ therefore wished to determine the prevalence and patterns of alcohol and drug usage in an applicable trauma population in our department. Only then could we rationally discuss the

Table 1 Toxicology studies in trauma populations

\begin{tabular}{|c|c|c|}
\hline Toxicological study & Alcohol prevalence & Drug prevalence \\
\hline Trauma and altered mental status (Chicago, $1988^{12}$ ) & $27 \%>100 \mathrm{mg} / \mathrm{dl}$ & $\begin{array}{l}37 \% \text { Cannabinoids, } 34 \% \text { cocaine, } 16 \% \text { opiates, } 7 \% \\
\text { benzodiazepines, } 2 \% \text { amphetamines }\end{array}$ \\
\hline Major trauma admissions (Cleveland, $1989^{10}$ ) & $63 \%$ Alcohol positive, no limit given & $\begin{array}{l}42 \% \text { Cocaine, } 8 \% \text { benzodiazepines, } 7 \% \text { opiates, } 7 \% \\
\text { cannabinoids }\end{array}$ \\
\hline Major trauma (Seattle, $1988^{11}$ ) & $31 \%>100 \mathrm{mg} / \mathrm{dl}$ & $\begin{array}{l}26 \% \text { Cannabinoids, } 12 \% \text { opiates, } 10 \% \text { cocaine, } 4 \% \\
\text { benzodiazepines }\end{array}$ \\
\hline Adolescent trauma (Philadelphia, $1992^{15}$ ) & $13 \%$ Alcohol positive, no limit given & $8 \%$ Cocaine, $6 \%$ cannabinoids, $6 \%$ diazepam, $6 \%$ opiates \\
\hline Seriously injured trauma admissions (Baltimore, $1996^{16}$ ) & $32 \%$ Alcohol positive, no limit given & $\begin{array}{l}15 \% \text { Opiates, } 14 \% \text { cocaine, } 11 \% \text { cannabinoids, } 0.1 \% \\
\text { amphetamines }\end{array}$ \\
\hline
\end{tabular}




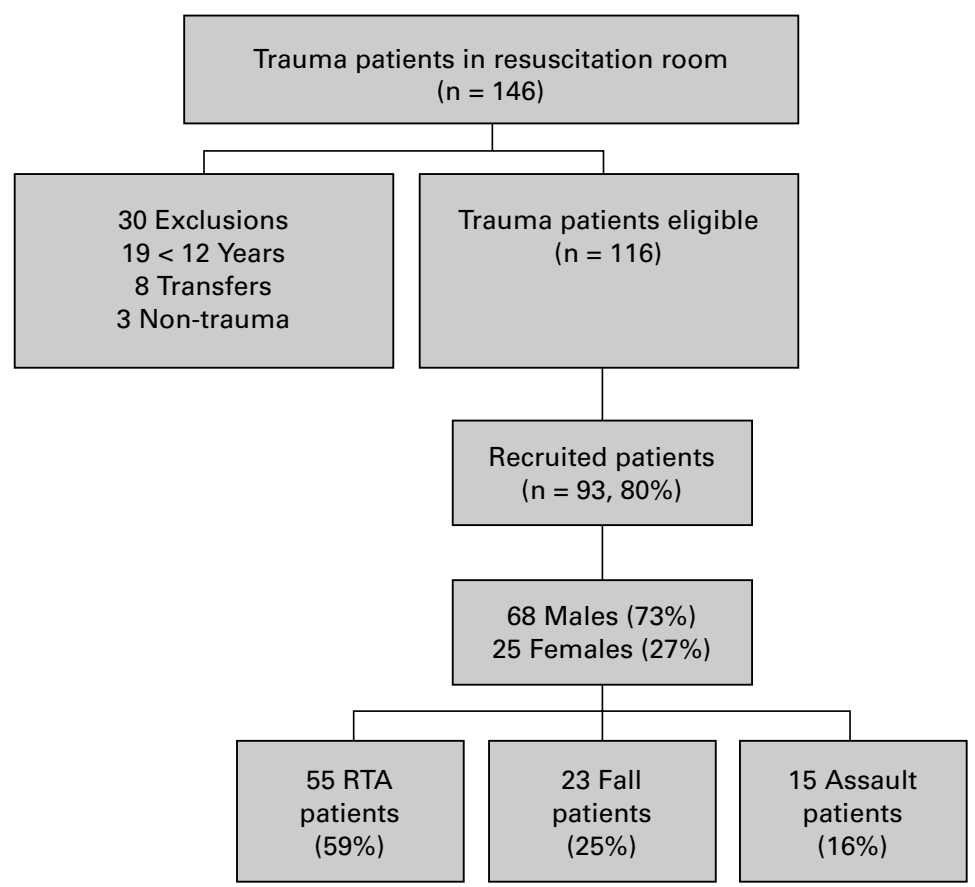

Figure 1 Patient enrolment data ( $R T A=$ road traffic accident $)$.

role of our department in tackling drug misuse, although treatment and education of the individual is not the only way forward, as recently emphasised in the Home Office report on drug misuse and the environment. ${ }^{18}$

\section{Methods}

STUDY DESIGN

This was a prospective hospital based prevalence study, at St James's University Hospital in Leeds, West Yorkshire. The A\&E department sees approximately 95000 patients per year, with approximately 300 patients being victims of significant trauma.

Inclusion criterion-All patients greater than 12 years of age, who were being assessed and treated for trauma related injuries in the resuscitation room of St James's University Hospital emergency department, were included.

Exclusion criteria-Trauma patients less than 12 years of age, tertiary referrals from outside St James's Hospital catchment area, and non-traumatic presentations to the resuscitation room were excluded.

When samples were taken for routine analysis and cross match, an anonymised bar coded plasma ethanol and urinary sample (when available) was taken and sent to the chemical pathology laboratory for appropriate refrigeration and analysis the next working day. Additional data added to the matching bar coded request form were anonymous and nonspecific to the patient-that is, sex (male or female), age band (12-17 or 18 and above), and mechanism of injury (RTA or non-RTA, which were falls and assaults).

Ethanol estimation was quantitatively performed using an enzymatic assay (Sigma Chemical Company). A legally positive alcohol screen was determined to be a concentration above $80 \mathrm{mg} / \mathrm{dl}$, which is the current legal driving limit for blood alcohol in the UK.
Table 2 Plasma ethanol results: subgroup analysis

\begin{tabular}{lcll}
\hline Patient group & $\begin{array}{l}\text { Patients }>80 \\
\text { mg/dl } \\
\text { (subgroup } \\
\text { total) }\end{array}$ & $\begin{array}{l}\text { Group } \\
\text { prevalence } \\
(\%)\end{array}$ & $\begin{array}{l}\text { 95\% } \\
\text { Confidence } \\
\text { intervals } \\
\text { (\%) }\end{array}$ \\
\hline RTA & $9(52)$ & 17 & 7 to 27 \\
Non-RTA & $15(37)$ & 41 & 25 to 57 \\
Fall & $9(23)$ & 39 & 19 to 59 \\
Assault & $6(14)$ & 43 & 17 to 69 \\
Male & $19(66)$ & 29 & 18 to 40 \\
Female & $5(23)$ & 22 & 5 to 39 \\
Adolescent & $1(10)$ & 10 & - \\
Total & $24(89)$ & 27 & 18 to 36
\end{tabular}

RTA $=$ road traffic accident.

Comprehensive urine toxicological analysis was initially by immunoassay (EMIT, SyvaUK), which qualitatively detected the presence of opiates, amphetamines, benzodiazepines, cannabinoids, cocaine, and methadone. Screening tests were deemed either positive or negative. Second line testing, using thin layer and gas chromatography/mass spectrometry, was used to specify and confirm the drug or metabolite involved, and therefore a drug of abuse, such as diacetylmorphine or amphetamine, could be distinguished from drugs of treatment such as codeine or pseudoephedrine. To exclude iatrogenic drugs from positive results, a positive opiate confirmation was married with the opiate administered in the resuscitation room (only the lead investigator had access to this information). Thus any specific opiate detected that matched the opiate given was excluded from the final confirmed results.

The study lasted six months, from 1 July to 31 December 1997, aiming for a recruitment of approximately 150 patients. The data were divided into three categories by mechanism of injury: RTA, fall, and assault.

95\% Confidence intervals were used for reporting of prevalence results, and the proportions test was used to compare prevalence results among the mechanism of injury and gender groups.

ETHICS

St James's Trust Clinical Research Committee granted approval to the study, provided that no additional invasive intervention was performed on the patient, and that acquisition of a urine sample and serum sample was not detrimental to the care of the trauma patient.

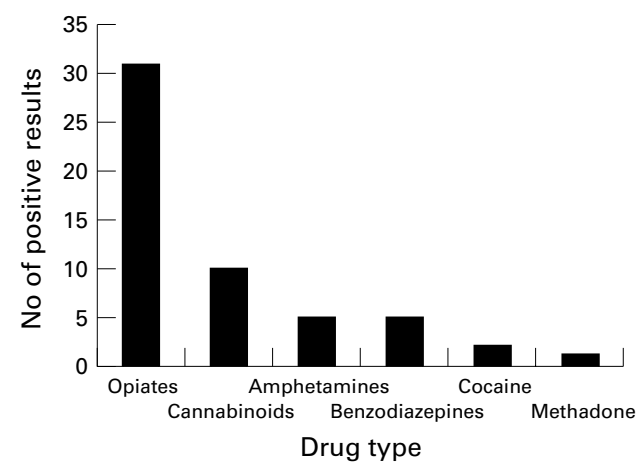

Figure 2 Immunoassay screening results. 


\section{Results}

PATIENT ENROLMENT

A total of 146 trauma patients were seen in the resuscitation room in the six month period, according to the resuscitation room log book and the local major trauma outcome study database. There were 30 exclusions, and the patient enrolment data is shown in fig 1 .

\section{PLASMA ETHANOL SCREENING}

Ethanol concentrations were measured on 89 samples. Of those, 52 were patients who had been involved in a RTA and 37 were non-RTA patients (three RTA patients and one non-RTA patient did not have a sample for analysis). Gender breakdown was 66 males and 23 females. Using $80 \mathrm{mg} / \mathrm{dl}$ as the legal limit, the results are displayed in table 2 .

In summary, there was a $27 \%$ prevalence rate of legally drunk patients in this population. Using the proportions test and 95\% confidence intervals, there was a significantly larger prevalence in the non-RTA group compared with the RTA group. No statistical difference was found between the RTA and the assault and fall groups $(p<0.05)$, nor between gender groups.

\section{TOXICOLOGICAL SCREENING}

Of the 93 patients entered, there were 79 adequate urine specimens collected, excluding two samples being of insufficient volume, one grossly bloodstained, and 11 having no urine sample available in the resuscitation room. Gender breakdown was 57 males and 22 females.

Preliminary immunoassay screening of those 79 samples revealed 40 patients with a positive toxicological screen, with a prevalence therefore of $51 \%$. Of these 40 samples, there were 20 positive for opiates alone, 11 patients positive for opiates and another drug, and nine with evidence of other drugs. Figure 2 is a graphical representation of the frequency of drug classes detected on initial screening.

Second line testing, utilising gas and thin layer chromatography, was therefore performed to specify the drug involved.

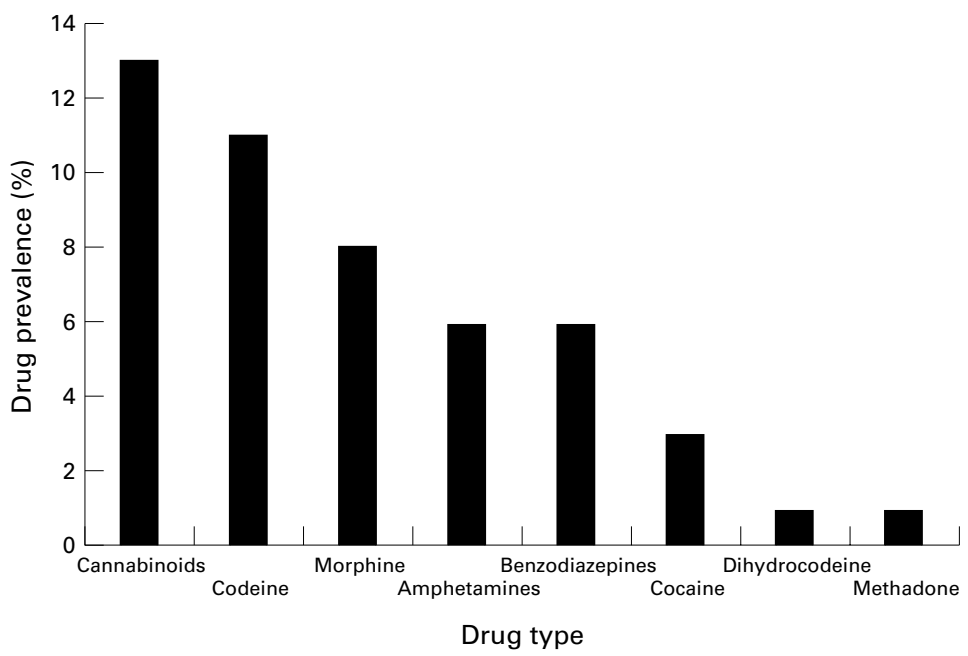

Figure 3 Chromatography results.
Of the 20 samples that screened positive for opiate alone, 11 samples were excluded due to the patient receiving intravenous morphine in the resuscitation room and subsequent detection of morphine via chromatography. Of the 11 samples that screened positive for opiates and other drugs, morphine was administered and detected in six samples, leaving five samples confirmed to have a specific opiate in combination with another drug.

Therefore, with respect to opiate positive screens, chromatography confirmed nine patients positive for codeine, with four of these in combination with another druge (dihydrocodeine, amphetamine, cannabinoid, and cocaine respectively). Six patients were confirmed positive for morphine (three were mixed, one in combination with amphetamine, one with methadone, and one with a cannabinoid), and one patient was confirmed positive for dihydrocodeine (in combination with codeine).

The 10 cannabinoid positive screens were all confirmed to have tetrahydrocannabinol metabolites present, and three of these were in combination with codeine, amphetamine, and morphine respectively.

All five amphetamine positive screens were confirmed on thin layer chromatography to be positive for amphetamine only, with no evidence of MDMA (ecstasy), methylamphetamine, phentermine, ephedrine, pseudoephedrine, or phenylpropanolamine. In three samples, amphetamine was accompanied by morphine, codeine, and a cannabinoid respectively.

Of the five benzodiazepine positive screens, one sample had both oxazepam and nordiazepam, two screens were in combination with cocaine metabolite, and one was mixed with methadone.

The presence of benzoylecgonine, the major metabolite of cocaine, was confirmed in the two cocaine positive samples by chromatography, and both were in combination with a benzodiazepine. Methadone and its major metabolite were detected in the methadone positive screen, found in combination with morphine.

The individual drug prevalence results for this trauma population are graphically shown in fig 3, and the subgroup analysis of positive chromatography results are displayed in table 3.

In summary, there were 28 patients with at least one positive drug confirmation on chromatographic analysis, which gives a prevalence of $35 \%$ in this trauma population for drug usage. Using proportion testing, no significant

Table 3 Urine chromatography results: subgroup analysis

\begin{tabular}{lcll}
\hline Patient group & $\begin{array}{l}\text { Confirmed } \\
\text { positive results } \\
\text { (subgroup total) }\end{array}$ & $\begin{array}{l}\text { Group } \\
\text { prevalence } \\
\text { (\%) }\end{array}$ & $\begin{array}{l}\text { 95\% } \\
\text { Confidence } \\
\text { intervals } \\
\text { (\%) }\end{array}$ \\
\hline RTA & $17(47)$ & 36 & 22 to 50 \\
Non-RTA & $11(32)$ & 34 & 18 to 50 \\
Fall & $5(21)$ & 24 & 6 to 42 \\
Assault & $6(11)$ & 55 & 26 to 84 \\
Male & $22(57)$ & 39 & 26 to 52 \\
Female & $6(22)$ & 27 & 8 to 46 \\
Adolescent & $1(10)$ & 10 & - \\
Total & $28(79)$ & 35 & 24 to 46 \\
\hline
\end{tabular}

RTA $=$ road traffic accident. 
differences were found between gender groups or mechanism of injury groups.

\section{Discussion}

From the plasma ethanol results it can be seen that there is a high prevalence of legally drunk or intoxicated patients in this British trauma population. There is a significantly higher prevalence in the non-RTA group compared with the RTA group. Our figures compare with the reported prevalence figures in table 1, but the study populations are varied and the legal limit (>100 mg/dl) was different. Other studies quoted did not specify the prevalence of patients over the legal limit.

With regard to toxicological screening for drugs, the confirmed drug prevalence of 35\% is high, and is so in all mechanisms of injury. The individual drug prevalence rates in our study reflect a different pattern of drug culture to studies in the United States, with less cocaine usage but similar use of cannabinoids. No attempt was made to compare proportions here because of the differing populations studied. It must also be remembered that these positive drug results are not proof of abuse and/or dependence. Detection of a drug in urine is also dependent on many factors including the size of dose, method of delivery, rate of drug metabolism, concentration of urine, fluid intake, and the physical condition of the patient. Table 4 shows the locally accepted detection time ranges for drugs in urine. Such variables make it difficult to specify the time of usage, and to be more specific, one would need serum or saliva sampling, which requires increased expertise and costs in detecting lower concentrations of drugs in these samples.

Drugs are not always taken in isolation, and our study revealed that three of five benzodiazepine positive samples and three of 10 cannabinoid positive samples were in combination with central nervous system stimulants or opiates. This highlights the complexity of toxicological influences in trauma, and while no individual causal link to trauma can be assumed, there appears to be an association that should not be discounted.

Ethical issues must also be addressed, and while there are no arguments about the identification of the drunk or drugged driver, there must be justification for screening trauma patients not involved in a RTA. An ethically acceptable solution may be to screen via

Table 4 Drugs and detection times in urine

\begin{tabular}{ll}
\hline Drug & $\begin{array}{l}\text { Approximate } \\
\text { detection time }\end{array}$ \\
\hline Opiates & 2 Days \\
Amphetamines & 2 Days \\
Cocaine metabolites & $2-4$ Days \\
Cannabinoids & 5 Days \\
$\quad$ Moderate use & 10 Days \\
Heavy use & 20 Days \\
Chronic smoker & 3 Days \\
Benzodiazepines & 4-6 Weeks \\
$\quad$ Therapeutic dosage & 3 Days \\
Methadone dosage & \\
\hline
\end{tabular}

$\star$ Reproduced with permission from Leeds Teaching Hospitals Toxicology Department. immunoassay, realising the limitations of substance sensitivity, and to confirm substance abuse and/or dependence via validated nonconfrontational questionnaires, which have been used to detect alcohol abuse or dependence in emergency populations. ${ }^{19}$ Soderstrom et al have estimated the prevalence of alcohol and drug abuse and dependency via validated structured clinical interviews, reporting that $24 \%$ of trauma patients were alcohol dependent and $18 \%$ were dependent on other drugs. ${ }^{16}$ They suggest patients with a positive toxicology screen and/or a positive screening questionnaire should be referred for formal evaluation and treatment.

Ongoing management may then involve brief advice protocols, which have recently been positively appraised, ${ }^{20}$ and have improved outcomes for at risk drinkers in the community. Further research is needed to see if brief advice protocols are applicable in the $A \& E$ department, and if they can influence other drug abuse behaviour.

Our hope is that drug screening is not perceived to be simply a means of prosecution, but rather a means for assessment of reversible drug abuse and/or dependence. Our study has shown that there is a notable prevalence of drug and alcohol use in this British A\&E trauma population. Rational discussion about the place of drug screening in our department can now take place. We also await further developments in brief counselling management to complement such screening, and suggest that the $\mathrm{A} \& \mathrm{E}$ department can become more active in initiating preventive medicine with the appropriate links to drug counselling services.

We thank the Leeds Teaching Hospitals Research and Development departments for their administrative and statistical assistment departments for their administrative and statistical assist-
ance and Dr J Barth and the Leeds General Infirmary chemical ance and Dr J Barth and the Leeds General Infirmary chemical
pathology staff involved in the facilitation of toxicological analypathology staff involved in the facilitation of toxicological analy-
sis. We also thank the doctors and nurses at St James's A\&E sis. We also thank the doctors and nurses at St James's A\&E
department who reminded each other to collect the necessary specimens.

Conflict of interest: none.

Funding: The St James's Special Trustees provided funding to meet the additional laboratory costs of chromatographic analyses.

\section{Contributor}

Thomas Carrigan initiated the study hypothesis, obtained ethics committee consent, formulated the protocol for specimen and data collection, and was involved in the analysis of results writing of the paper. Helen Field discussed core biochemical ideas, developed the laboratory protocol for collection and biochemical analysis of the specimens obtained, participated in the data analysis, and helped with editing of the paper from a toxicological perspective. Paul Gaffney participated in data collection, discussed core ideas, and edited the paper. Robin Illingworth discussed core ideas in developing the study design, participated in data collection, and helped with editing of the paper. Wayne in data collection, and helped with editing of the paper. Wayne Hamer helped with the study design and ethics committee

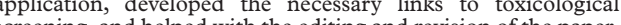
Thomas Carrigan is the guarantor for the paper.

1 Seppala T, Linnoila M, Mattila MJ. Drugs, alcohol and driving. Drugs 1979;17:389-408.

2 Resnick RB, Kestenbaum RS, Schwartz LK. Acute systemic effects of cocaine in man. Science 1977;195:696-8.

3 Rafaelsenos J, Beck P, Christiansen J, et al. Cannabis and alcohol: effects on simulated car driving. Science 1973;179: $920-3$.

4 Drummer OH. Drugs and accident risk in fatally injured drivers. Proceedings of the 13th international conference on alcohol, drugs, and traffic safety. Adelaide 1995;1:426-9.

5 Cremona A. Mad drivers: psychiatric illness and driving performance. Br f Hosp Med 1986;35:193-5.

6 Lerformanstein SR, Weissberg MP, Terry D. Alcohol intoxication, injuries, and dangerous behaviors. F Trauma 1990;30: $1252-8$ 
7 Rivara FP, Koepsell TD, Jurkovich GJ, et al. The effects of alcohol abuse on readmission from trauma. $7 A M A$ alcohol abuse on

8 Brewer RD, Morris PD, Cole TB, et al. The risk of dying in alcohol related automobile crashes among habitual drunk drivers. N Engl f Med 1994;331:513-17.

9 Maull KI, Kinning LS, Hickman JK. Culpability and accountability of hospitalised injured alcohol-impaired drivers. $7 A M A$ 1984;252:1880-3.

10 Parran TV, Weber E, Tasse J, et al. Mandatory toxicology testing and chemical dependence consultation follow-up in a level-one trauma center. F Trauma 1995;38:278-80.

11 Rivara FP, Mueller BA, Fligner CL, et al. Drug use in trauma victims. F Trauma 1989;29:462-70.

12 Sloan EP, Zalenski RJ, Smith RF, et al. Drug prevalence and its relationship to trauma severity and management. $f$ Trauma $1989 ; 29: 1647-53$.

13 Brookoff D, Cook CS, Williams C, et al. Testing reckless drivers for cocaine and marijuana. N Engl f Med 1994;331: 518-22.
14 Everest JT, Turnbridge RJ, Widdop B. The incidence of drugs in road traffic fatalities. TRL research report 202. Crowthorne, Berkshire: 1989 (RG116AU)

5 Loiselle JM, Baker MD, Templeton JM Jr, et al. Substance abuse in adolescent trauma. Ann Emerg Med 1993;22 1530-4.

16 Soderstrom CA, Smith GS, Dischinger PC, et al. Psychoactive substance abuse disorders among seriously injured trauma center patients. $\mathcal{F A M A} 1997 ; 277: 1769-74$.

17 Soderstrom CA, Dailey JT, Kerns TJ. Alcohol and other drugs: an assessment of testing and clinical practices in US trauma centers. F Trauma 1994;36:68-73.

18 Department of Health. Drug misuse and the environment. A report by the advisory council on the misuse of drugs. London: HMSO, 1998.

19 Cherpitel C. Screening for alcohol problems in the emergency department. Ann Emerg Med 1995;26:158-66.

20 Fleming MF, Lawton Barry K, Baier Manwell L, et al. Brief physician advice for problem alcohol drinkers. $\mathscr{F} A M A$ 1997;277:1039-45. 